\title{
First observation of thermospheric neutral wind at Chinese Yellow River Station in Ny-Ålesund, Svalbard
}

\author{
ZHANG Hong ${ }^{*}$, AI Yong*, ZHANG YanGe \& HU GuoYuan \\ School of Electronic Information, Wuhan University, Wuhan 430072, China
}

Received August 14, 2012; accepted November 8, 2012; published online December 28, 2012

\begin{abstract}
A compact and cost-effective all-sky Fabry-Perot interferometer, which is produced by Wuhan University and used for the observation of the thermospheric neutral wind, was installed at Chinese Yellow River Station in Ny-Ålesund, Svalbard in November 2010, and continuously operated in last two winter seasons. The 92-day all-sky interference data acquired from November 1, 2011 to February 26, 2012 were collected to get the velocity of thermospheric neutral wind, which was calculated from the Doppler shift caused by the movement of oxygen atom at the different layers. The database was divided into two periods: (1) The OI 557.7 $\mathrm{nm}$ emission was observed from November 1, 2011 to January 12, 2012. Observations showed that the velocity of horizontal wind is normally less than $\sim 40 \mathrm{~m} / \mathrm{s}$ on the quiet condition, and exceeded $100 \mathrm{~m} / \mathrm{s}$ on the disturbed condition; and (2) the OI $630.0 \mathrm{~nm}$ emission was observed from January 13, 2012 to February 26, 2012. Observations showed that the velocity of horizontal wind is normally less than $200 \mathrm{~m} / \mathrm{s}$, and enhanced to over $300 \mathrm{~m} / \mathrm{s}$ on strong magnetic activities. It shows that the velocities of meridional and zonal wind are more consistent with the velocities calculated from the model HWM07 at the higher layer, especially for the zonal direction at nightside auroral regions. Ion drag and Joule heating were the two important processes considered in the analysis of the relationship between the wind pattern and aurora, suggesting that wind speed would be increased and accelerated in the direction perpendicular to the aurora arc when the aurora activity becomes strong.
\end{abstract}

Fabry-Perot interferometer, thermosphere, winds, polar region, aurora

Citation: Zhang H, Ai Y, Zhang Y G, et al. First observation of thermospheric neutral wind at Chinese Yellow River Station in Ny-Ålesund, Svalbard. Chin Sci Bull, 2013, 58: 1310-1315, doi: 10.1007/s11434-012-5626-9

The thermosphere is the fourth layer of the Earth's atmosphere. It can not only get energy and momentum from the lower layer (i.e. the mesosphere) by the gravity wave and tide [1-3], but also from the solar wind through the magnetosphere [4]. Neutral particles are the main components in the thermosphere, where neutral wind is coupled with ion flow for a long time, especially in the polar region. The thermosphere is where wind-induced horizontal current diverge and the neutrals can influence the magnetospheric convection [5]. If the neutral wind effect is neglected, especially below $100 \mathrm{~km}$ altitude, some errors may occur when the atmosphere dynamics is studied [6]. Neutral wind is also important in investigating the generation of polar cap sporadic $\mathrm{E}$, and the wind shears mechanism is a possible spo-

*Corresponding authors (email: Rainbow.313@163.com; aiyong09@163.com) radic E layer mechanism [7]. Brekke et al. [8] suggested that for a true heat estimate input to the neutral upper atmosphere caused by ionospheric currents driven by the magnetospheric and neutral wind dynamos, a good estimate of neutral wind vector is fundamentally important.

For many years, the Fabry-Perot interferometer has been an effective equipment for the observation of thermosphere neutral winds [9-13]. In China, a three-channeled FabryPerot interferometer was deployed at the Chinese Xinglong Station in 2010 [14]. A two-channeled Fabry-Perot interferometer is operated at another Chinese station in Hebei Province. Both interferometers are for mid-latitude observation. In the winter of 2010, a compact and cost-effective all-sky Fabry-Perot interferometer (WD10-ASFPI) produced by Wuhan University was installed at Chinese Yellow River Station in Ny-Ålesund, Svalbard for the observa- 
tion of thermospheric wind in polar region.

\section{Equipment}

The Chinese Yellow River Station (YRS) at Ny-Ålesund, Svalbard is located at geographic coordinates $78.92^{\circ} \mathrm{N}$, $11.93^{\circ} \mathrm{E}$ and corrected geomagnetic latitude $76.24^{\circ}$, MLT $\sim \mathrm{UT}+3 \mathrm{~h}$. During the long period of polar night (from November to February of the next year), the optical instruments at YRS can make almost $24 \mathrm{~h}$ surveys of optical observations [15]. The all-sky Fabry-Perot interferometer was first transported to the YRS in November 2010 from Wuhan University, upon which it began observation of the thermosphere after several adjustments. The simple configuration is shown in Figure 1(a). Figure 1(b) shows the observation house environment from the roof of the YRS.

Dome is optically and highly transparent to allow vast amounts of light to go through. The fisheye lens is Nikon AF DX Fisheye-Nikkor $10.5 \mathrm{~mm} \mathrm{f/2.8G} \mathrm{ED,} \mathrm{with} \mathrm{an} \mathrm{almost}$ $180^{\circ}$ field of view (FOV). The filters are for different wavelengths ( $557.7 \mathrm{~nm}$ for green, $630.0 \mathrm{~nm}$ for red). The most important element is the Fabry-Perot etalon, which is a solid etalon with refractive indices of fused silica, 1.459592 and 1.457012 , for wavelengths 557.7 and $630.0 \mathrm{~nm}$, respectively. The reflector coating is greater than $90 \%$ and the parallel plates are $15 \mathrm{~mm}$ thick on both wavelengths. CCD is a scientific camera from Andor Company, namely, the iKon-M cooled CCD camera with $1024 \times 1024$ pixels, which benefits from negligible dark current with industry-leading thermoelectric cooling down mechanism, enabling the use of significantly longer exposure times.

Although the fisheye lens has a nearly $180^{\circ} \mathrm{FOV}$, the available FOV of the FPI is only $150^{\circ}$ because of the limitation of other elements in the optical path. The CCD was binned $2 \times 2$ on chip to produce $512 \times 512$-pixel images at a rate of $2.5 \mathrm{MHz}$ for both wavelengths. The FPI can obtain more than eight-order Interference circles, only the inner

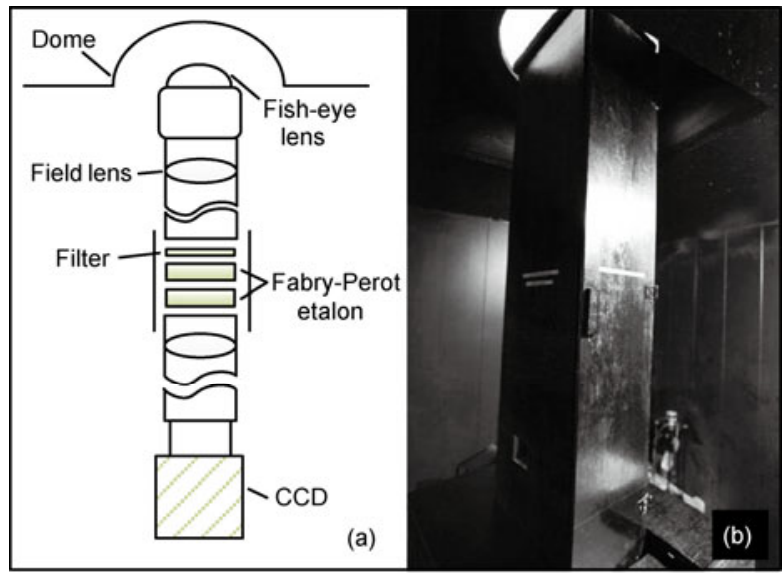

Figure 1 (a) Simple configuration of an all-sky Fabry-Perot interferometer; (b) the observation house environment. five circles, however, are chosen to inverse the neutral LOS velocity because there is a distortion on the edge of the images. The radius of the observation circle in the sky depends on the height of the observed light source and the location of the fringe in the image data.

The neutral wind observation during the winter of 2011 at YRS was carried out from November 1, 2011 to February 26,2012 , including two different periods at different wavelength measurements. Before January 12, 2012, it was experimented for the $557.7 \mathrm{~nm}$, and then later operated for $630.0 \mathrm{~nm}$. The time resolution of the observational wind data was $8-10 \mathrm{~min}$ and the altitudes of the observation were assumed to be typically 110 and $240 \mathrm{~km}$ at 557.7 and 630.0 $\mathrm{nm}$, respectively.

\section{Observation results}

According to the World Data Center for Geomagnetism in the Kyoto website, we chose the quietest day (December 27, 2011) and most disturbed day (December 29, 2011) for $557.7 \mathrm{~nm}$ wavelength, the quietest day (January 19, 2012) and most disturbed day (January 23, 2012) for $630.0 \mathrm{~nm}$, respectively, with clear skies over the station. These international Q-Days and D-Days are derived from $K p$ index which are indicated in Figure 2 for the four days.

On the disturbed condition, the intensity of the images could be strengthened when the aurora occurred over the sky of station. Figure 3 shows the intensity of the interference images for these days. Each image is divided into 380 slices vertically as illustrated in Figure 3(a). The accumulation of the intensity of these slices on the four days is presented in Figure 3(b) which is also divided by the background emission. The bright color in Figure 3(b) indicates probably intensive aurora effect on the images during the geomagnetic activity.

The variation characteristics of meridional and zonal winds on four days are presented as follows. Considering

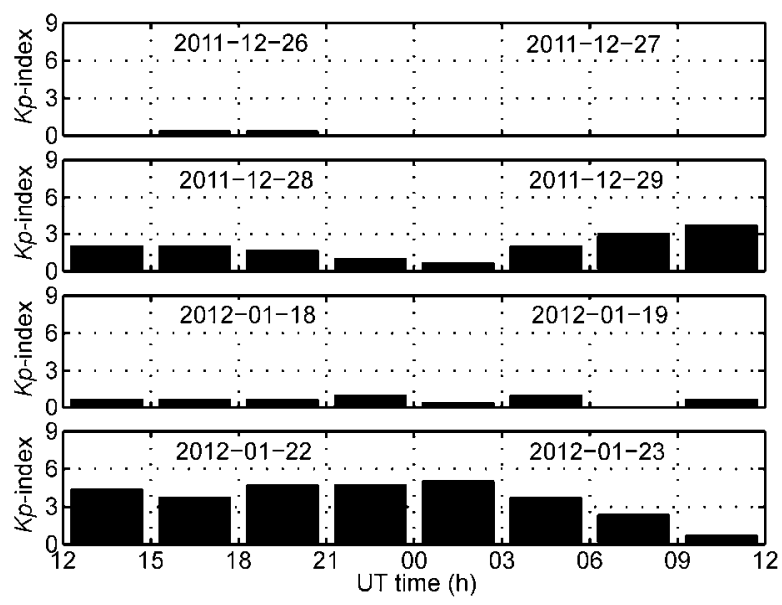

Figure $2 K p$ index for $4 \mathrm{~d}$. 


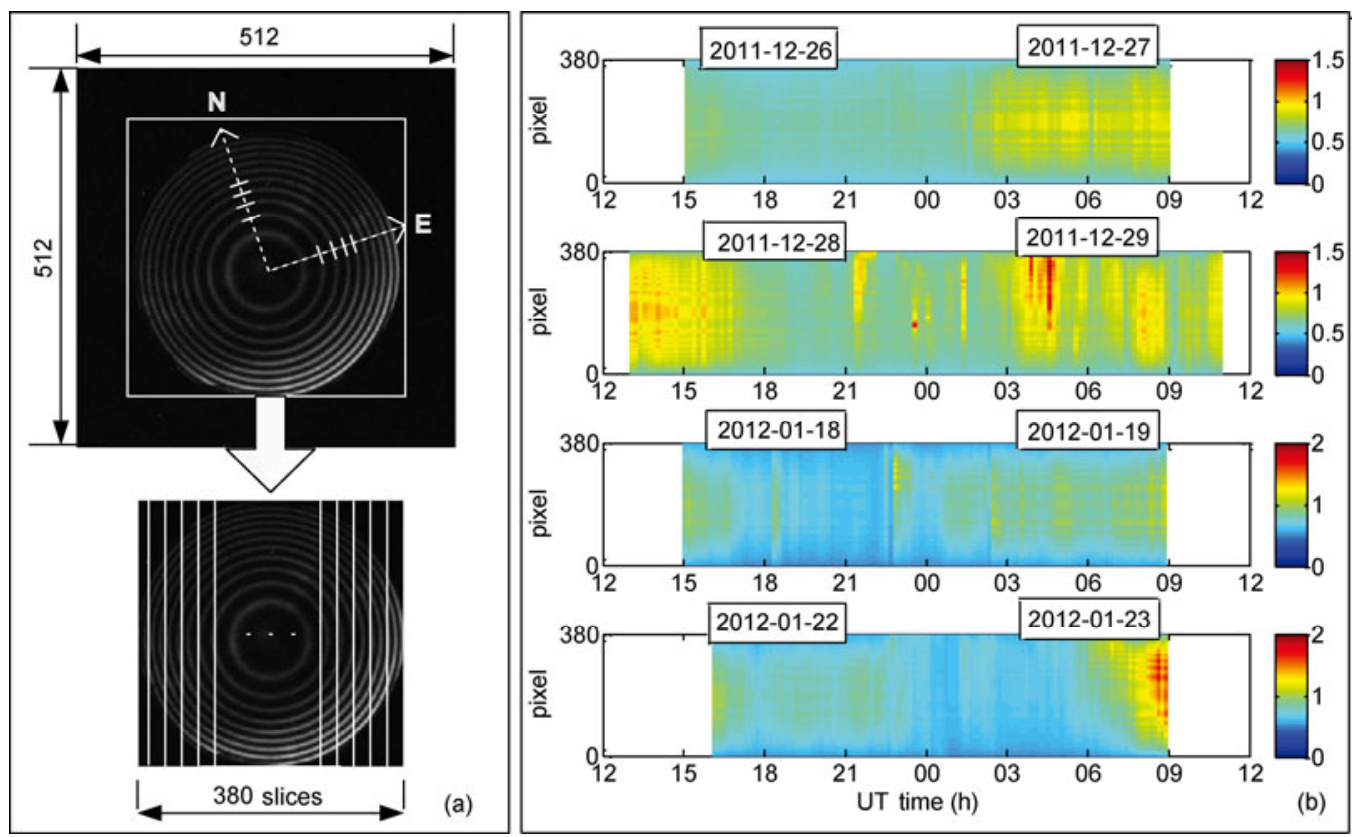

Figure 3 (a) Illustration for the 380 slices on interference image; (b) intensity of image for December 27 and 29, 2011, and January 19 and 23, 2012, respectively. The accumulation intensity is divided by the accumulation of background intensity.

the nonuniformity of the horizontal wind in the polar region, the north and east directions that were specifically chosen as meridional and zonal winds for the calculation of the horizontal wind from the LOS wind field, were then compared with the HWM07 model. The observational results of the calculation of the four winds from different order rings are indicated in Figure 3(a), with the short lines indicating the $\mathrm{N}$ (north) and $\mathrm{E}$ (east) directions. The inner order ring of the image with the small angle to the zenith $\left(\leqslant 15^{\circ}\right)$ was used to represent the vertical wind during the calculation of the horizontal wind to eliminate the effect of the vertical wind which is prominent in high latitudes.

\subsection{Event 1, 2: December 27 and 29, 2011}

Figure 4 exhibits the comparison of measured OI $557.7 \mathrm{~nm}$ meridional and zonal winds with the HWM07 model. Four colored curved lines represented the four different winds with their number in accordance to their distance away from the center of the image, as illustrated in Figure 3(a). The results from the HWM07 model (black dotted line) were calculated with parameters close to the location of the fourth ring (blue), given that the large angle to the zenith contributed more from the horizontal component for the outmost order ring. The peak amplitudes of the winds in Figure 4(a) and (b) were approximately $60 \mathrm{~m} / \mathrm{s}$ at midnight of December 27 at the meridional direction for the third wind, and $50 \mathrm{~m} / \mathrm{s}$ around 21:00 UT at the zonal direction for the third and fourth winds. However, the other winds were more stable during this day with approximately $40 \mathrm{~m} / \mathrm{s}$ for most periods. The discrepancies among the four winds were likely because of the location of the measured point with different latitude or longitude. The winds on December 29, 2011 in Figure 4(c) and (d) were more consistent compared with the winds on December 27, 2011 in Figure 4(a) and (b), particularly after midnight with increased amplitude. The meridional wind in Figure 4(c) exhibited the maximum amplitude of $123 \mathrm{~m} / \mathrm{s}$ during the 00:00 UT to 03:00 UT, after which it decreased. The disorder of the winds at premidnight was probably because of their locations, whereas the consistent trend at post-night was probably because of the force during the geomagnetic activity. The results of HWM07 presented clear discrepancies to the observed results, and were not sensitive to geomagnetic activity.

\subsection{Event 3, 4: January 19 and 23, 2012}

Figure 5 has the same format as in Figure 4. It is the image taken at the layer of OI $630.0 \mathrm{~nm}$ on January 19 and 23, 2012. Some data were missing because of the noise on the images. The winds from the four order rings in Figure 5(a), (b), and (d) were in very close agreement with each other, and the wind from the outmost ring (blue) exhibited a consistent phase with HWM07 results, except for some differences with the amplitude. However, the wind shears are shown in Figure 5(c) around 21:00 UT, 01:00 UT, and 05:00 UT with the disturbed geomagnetic condition. Moreover, a strong northward (polarward) wind arose after midnight particularly for the first wind (red) in Figure 5(c) which was close to the station. The strong trend in the north probably followed the plasma convection at the local area with a highly variable energy input. The observed result has 

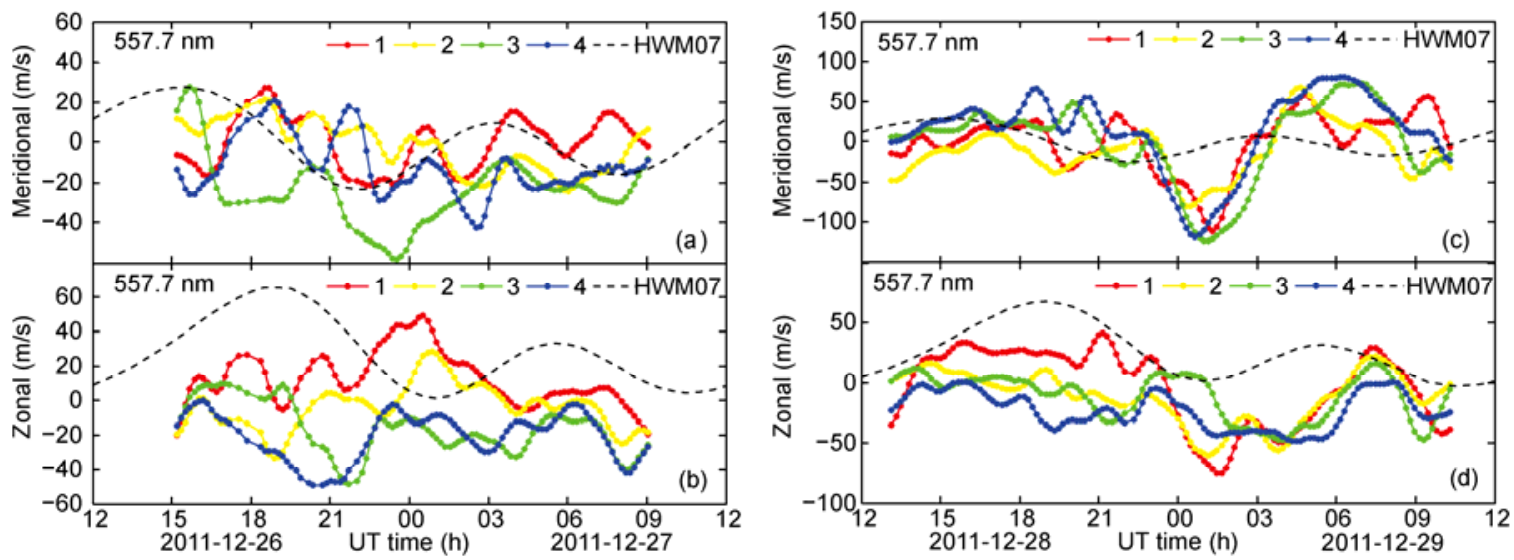

Figure 4 Wind velocity at $557.7 \mathrm{~nm}$ oxygen atom layer on December 27 (a), (b) and December 29 (c), (d), 2011. The colored lines symbolize the wind from different order rings. The black dotted line is the wind estimated from the HWM07 model. The east and north are the positive directions for the meridional and zonal wind, respectively.
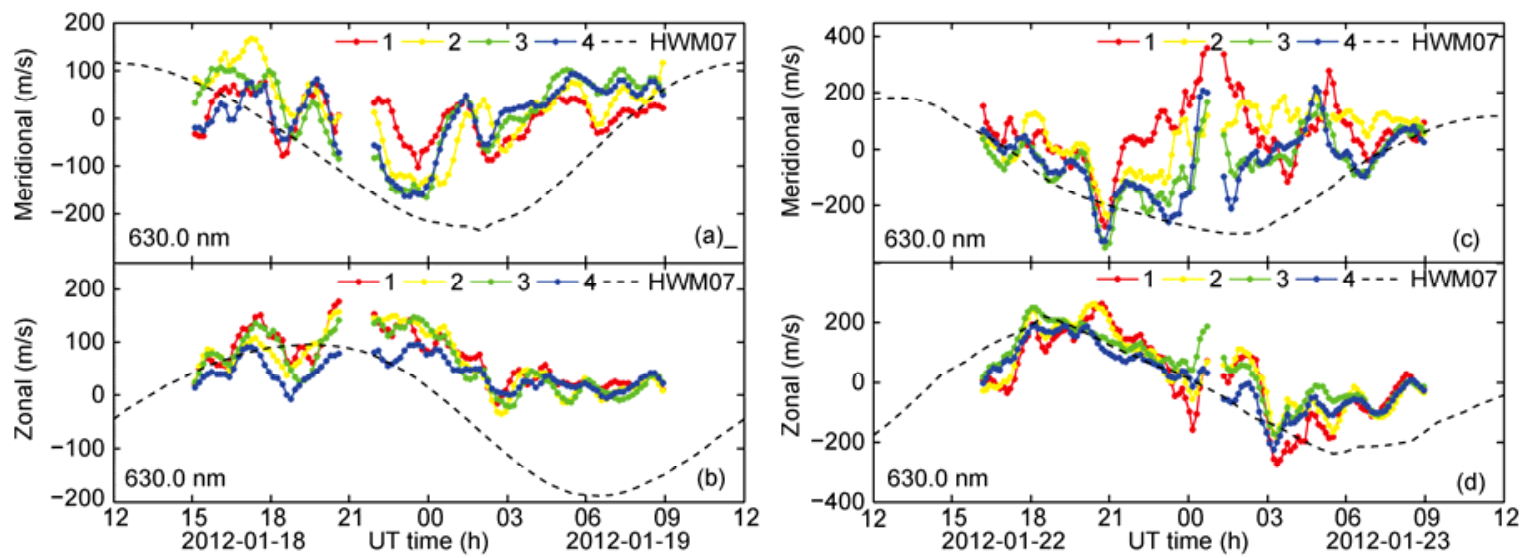

Figure 5 Wind velocity at the $630.0 \mathrm{~nm}$ oxygen atom layer on January 19 (a), (b) and January 23 (c), (d), 2012 . The format is the same as in Figure 4.

the most similar behavior with the model for the zonal direction at nightside auroral regions (15:00 UT to 03:00 UT) as shown in Figure 5(d). The amplitudes shown in Figure 5 (a) and (b) did not exceed $200 \mathrm{~m} / \mathrm{s}$, but a significantly increased wind that exceeded $300 \mathrm{~m} / \mathrm{s}$ was observed at around 01:00 UT on a disturbed day, as shown in Figure 5(c).

\section{Discussion}

In the polar region analysis, the thermospheric wind field that responded first to the aurora was discussed. Figure 4(a) and (b) presents the amplitude of the wind field less than 60 $\mathrm{m} / \mathrm{s}$, while at the disturbed condition as shown in Figure 4(c) and (d), neutral winds were greatly influenced by solar wind and relevant geomagnetic activity, shown by the $K p$ index in Figure 2 as well as some light colors from 03:00 UT to 09:00 UT on December 29. Figure 6 displays some LOS wind fields and the corresponding interference images for these two days during the period from 06:00 UT to 09:00
UT. The wind is expressed in the color map with a red and blue color for outward $100 \mathrm{~m} / \mathrm{s}$ and inward $100 \mathrm{~m} / \mathrm{s}$ positions from the center of the station, respectively.

The significant differences between the two different-condition days are shown in Figure 6. When there were no activities, the LOS wind field changed slowly. Thus, for the aurora appearing (indicated with the bright light in the interference images) southeast of the image, the wind would be accelerated in a perpendicular direction to the aurora arc almost northward (geographical), and the amplitude of the wind is obviously increased. Brändström [16] also stated that when a stable arc is seen, the neutral wind is mainly northward. A preliminary analysis of their dataset suggests that the wind is directed perpendicular to the arc, with very low wind speeds parallel to the arc.

Figure 7 exhibits the all-sky LOS velocity of OI 630.0 $\mathrm{nm}$ from 06:00 UT to 09:00 UT on January 19 and 23, 2012 with the same format as Figure 6. The relevance between the pattern of the LOS wind and the aurora activities can be clearly observed. Accelerated wind velocity can reach the maximum of $300 \mathrm{~m} / \mathrm{s}$ at some disturbed periods. 


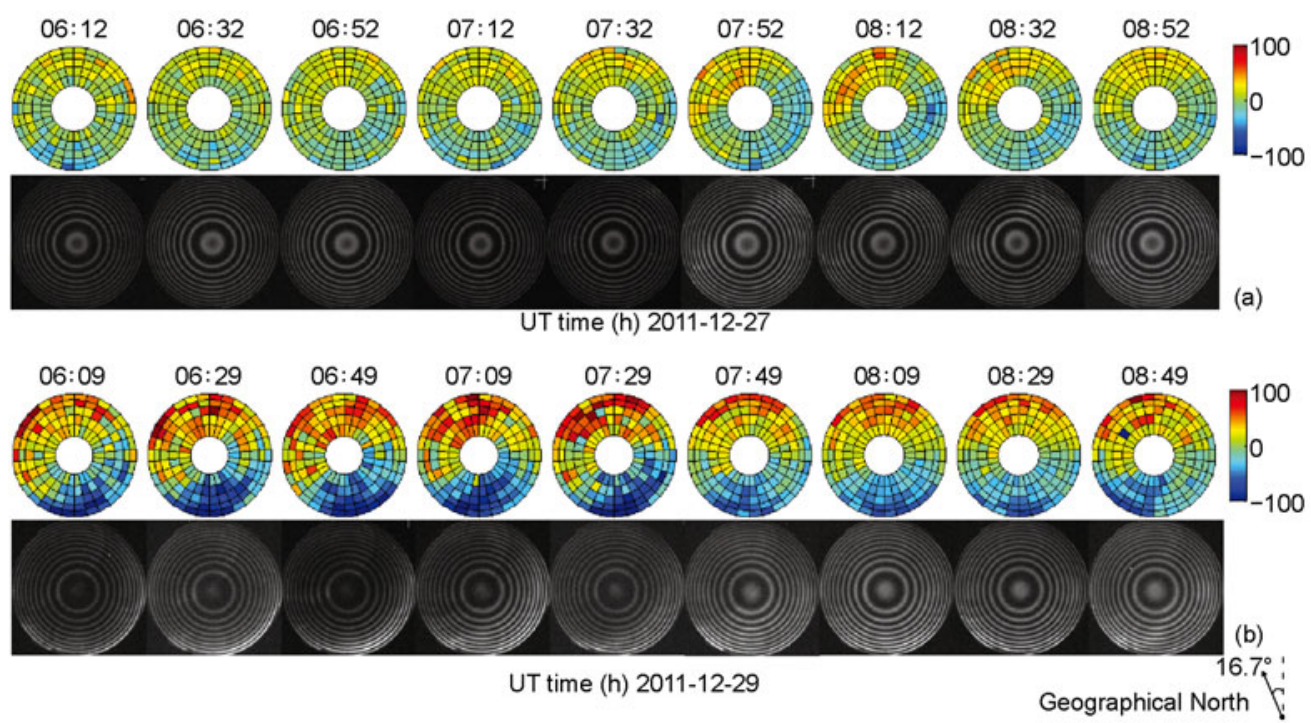

Figure 6 The all-sky LOS velocity of $557.7 \mathrm{~nm}$ from 06:00 UT to 09:00 UT on December 27 (a) and December 29 (b), 2011 . Red indicates the 100 m/s outward position and blue indicates the $100 \mathrm{~m} / \mathrm{s}$ inward position from the center of the station in the LOS direction. Geographical north is indicated at the bottom-right corner.
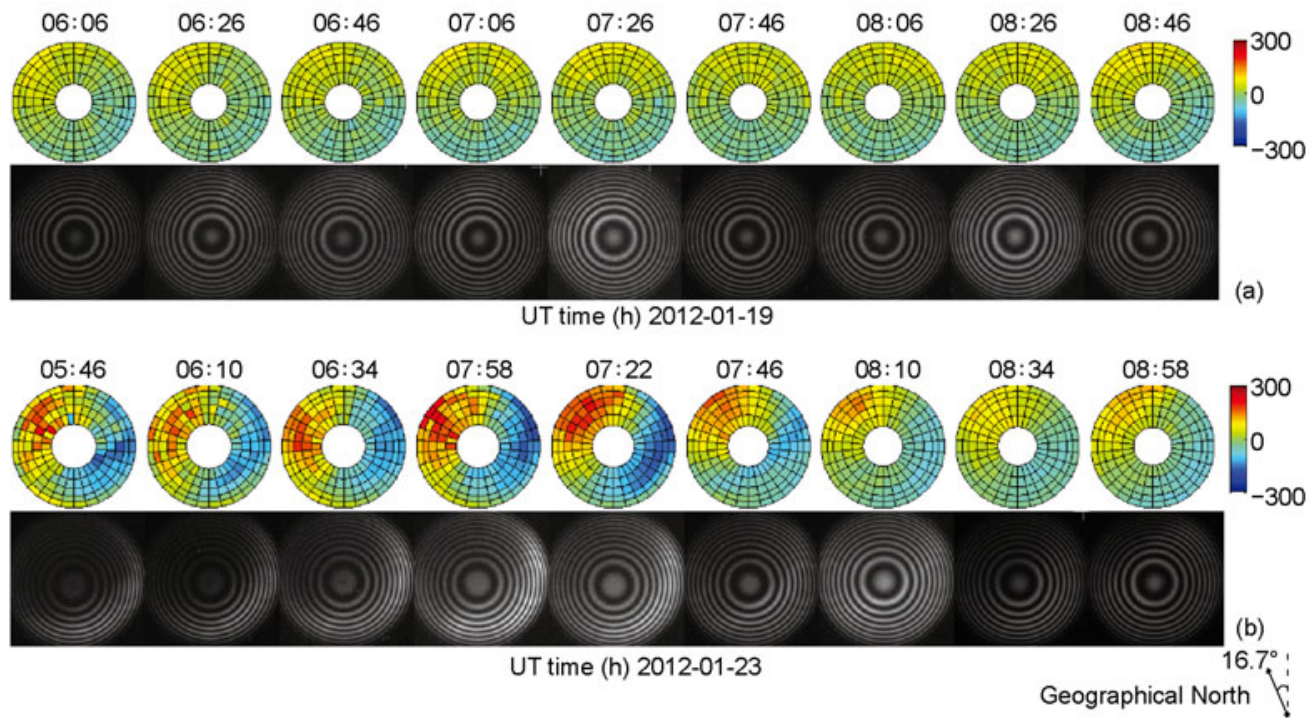

Figure 7 The all-sky LOS velocity at $630.0 \mathrm{~nm}$ from 06:00 UT to 09:00 UT with the same format as in Figure 6 on January 19 (a) and January 23 (b), 2012 , where color red indicates the $300 \mathrm{~m} / \mathrm{s}$ outward position and blue indicates the $300 \mathrm{~m} / \mathrm{s}$ inward position.

The enhanced direction is also perpendicular to the aurora arc, but weakens in the paralleled direction with the arc.

The two most important processes are discussed, which could possibly affect neutral wind, ion drag, and Joule heating. Tsuda et al. [17] mentioned that during ion drag, the ion motion induced by the convection electric field drives or modifies the neutral atmospheric motion through ion-neutral collisions, whereas during the Joule heating case, the pressure gradient is modified, which corresponds to the frictional heating between ions and neutrals. Wilson et al. [18] indicated that in the smaller storm heating events, the energy input is equally divided, with Joule heating slightly dominating over particle precipitation; in larger events,
Joule heating clearly dominates. Therefore, the activities of the ions will have much more influence on the pattern of the neutral wind during the disturbed condition.

At the altitude of $557.7 \mathrm{~nm}$ oxygen atom, the layer is mainly decided by the neutral wind when the electric field $\mathrm{E}$ is small. However, when the ionospheric E region density increases in response to the more intense auroral particle precipitation, the neutral wind may be driven by the ion velocity through the collision and will move toward the same direction. A significant enhancement of wind velocity is observed in Figure 6 when the aurora appeared. The particle precipitation is associated with the aurora, so the heating produced by the current could expand the neutral gas, 
causing the anti-aurora region flow.

Different from the $557.7 \mathrm{~nm}$ oxygen atom, the altitude of OI $630.0 \mathrm{~nm}$ almost belongs to the $\mathrm{F}$ region in the ionosphere, where there is high viscosity because of tenuous gas. Aruliah et al. [19] mentioned that the high viscosity soothes out small-scale structures. By comparison, the winds of OI $557.7 \mathrm{~nm}$ in Figure 4 behave in a more disorderly manner most of the time while winds of OI $630.0 \mathrm{~nm}$ in Figure 5 display a tendency for eight-hour cycles with good agreement. Compared with the charge particle, the neutral gas is much less at this layer, resulting in less ion-neutral collision. The ions dominate the pattern of wind and drag the neutral gas with it. When a large event happens, Joule heating dominates the energy distribution and makes the ions and neutral gas flow severely.

\section{Conclusion}

Observation of thermospheric neutral wind at Chinese Yellow River Station in Ny-Ålesund to obtain high-atmosphere data of the wind fields in the polar region is conducted for the first time. The result is presented with the meridional and zonal wind field for four days with two different wavelengths. The comparison of the wind between the different geomagnetic conditions indicates that the amplitudes of the wind are accelerated during the disturbed conditions with the aurora appearance. The analysis is focused on the performance of the wind pattern with the relationship to the aurora, which increases in the direction perpendicular to the aurora arc. Two important processes, ion drag and Joule heating, are the most possible reasons to explain the influence on the pattern of the wind field.

Further work will be concentrated on two aspects: (1) Improved precision by comparing the data with other equipment and correcting lens distortion to enable the use of more order rings, including the one near the edge of the image. (2) Location calibration in the all-sky image with the corresponding measured point in the sky and calculation of the horizontal wind field from the LOS wind field.

This work was supported by the National High-Tech R\&D Program of China and the Chinese Arctic and Antarctic Administration.

1 Hickey M P, Walterscheid R L, Schubert G. Gravity wave heating and cooling of the thermosphere: Sensible heat flux and viscous flux of kinetic energy. J Geophys Res, 2011, 116, doi:10.1029/2011JA
016792

2 Tu C, Hu X, Yan Z A, et al. First imaging observation of the gravity waves in the mesopause region in China. Chin Sci Bull, 2010, 55: 539-543

3 Jiang G Y, Xu J Y, Shi J K, et al. The first observation of the atmospheric tides in the mesosphere and lower thermosphere over Hainan, China. Chin Sci Bull, 2010, 55: 1059-1066

4 Burke W J. Solar cycle dependence of solar wind energy coupling to the thermosphere. J Geophys Res, 2011, 116, doi: 10.1029/2011JA 016437

5 Ridley A J, Richmond A D, Gombosi T I, et al. Ionospheric control of the magnetospheric configuration: Thermospheric neutral winds. J Geophys Res, 2003, 108, doi: 10.1029/2002JA009464

6 Sangalli L, Knudsen D J, Larsen M F, et al. Rocket-based measurements of ion velocity, neutral wind, and electric field in the collisional transition region of the auroral ionosphere. J Geophys Res, 2009, 114, doi: 10.1029/2008JA013757

7 Nygrén T, Voiculescu M, Aikio A T. The role of electric field and neutral wind in the generation of polar cap sporadic E. Ann Geophys, 2008, 26: 3757-3763

8 Brekke A, Nozawa S, Sparr T. Studies of the $E$ region neutral wind in the quiet auroral ionosphere. J Geophys Res, 1994, 99: 8801-8825

9 Rees D, Greenaway A H. Doppler imaging system; an optical device for measuring vector winds. 1: General principles. Appl Opt, 1983, 22: 1078-1083

10 Biondi M A, Sipler D P, Zipf M E, et al. All-sky Doppler interferometer for thermospheric dynamics studies. Appl Opt, 1995, 34: 16461654

11 Innis J L, Greet P A, Dyson P L, et al. Fabry-Perot spectrometer observations of the auroral oval/polar cap boundary above Mawson, Antarctica. J Atmos Terr Phys, 1996, 58: 1973-1988

12 Aruliah A L, Griffin E M, McWhirter I, et al. First tristatic studies of meso-scale ion-neutral dynamics and energetics in the high-latitude upper atmosphere using collocated FPIs and EISCAT radar. Geophys Res Lett, 2004, 31, doi: 10.1029/2003GL018469

13 Gerrard A J, Meriwether J W. Initial daytime and nighttime SOFDI observations of thermospheric winds from Fabry-Perot Doppler shift measurements of the 630-nm OI line-shape profile. Ann Geophys, 2011, 29: 1529-1536

14 Yuan W, Xu J Y, Ma R P, et al. First observation of mesospheric and thermospheric winds by a Fabry-Perot interferometer in China. Chin Sci Bull, 2010, 55: 4046-4051

15 Hu Z J, Yang H, Huang D, et al. Synoptic distribution of dayside aurora: Multiple-wavelength all-sky observation at Yellow River Station in Ny-Ålesund, Svalbard. J Atmos Sol Terr Phys, 2009, 71: 794-804

16 Brändström U. The Auroral Large Imaging System-Design, Operation and Scientific results. Kiruna: Swedish Institute of Space Physics, 2003. 108-110

17 Tsuda T T, Nozawa S, Oyama S, et al. Acceleration mechanism of high-speed neutral wind observed in the polar lower thermosphere. $\mathrm{J}$ Geophys Res, 2009, 114, doi: 10.1029/2008JA013867

18 Wilson G R, Weimer D R, Wise J O, et al. Response of the thermosphere to Joule heating and particle precipitation. J Geophys Res, 2006, 111, doi: 10.1029/2005JA011274

19 Aruliah A L, Griffin E M, Yiu H-C I, et al. SCANDI-An all-sky Doppler imager for studies of thermospheric spatial structure. Ann Geophys, 2010, 28: 549-567

Open Access This article is distributed under the terms of the Creative Commons Attribution License which permits any use, distribution, and reproduction in any medium, provided the original author(s) and source are credited. 